\title{
THALAMO-CORTICAL WAXING AND WANING NATURE AND ACTIVITIES OF CORTICAL NEURONS IN THE RABBIT
}

\author{
Takashi UCHIDA \\ Department of Physiology, Kyoto Prefectural \\ University of Medicine, Kyoto
}

There are two inconsistent interpretations concerning the origin of component potentials in the evoked potential obtained by stimulating the thalamic relay nucleus. The one is that the depolarization wave originating in action potentials of deep cortical cells ascends along their apical dendrites toward the surface and is recorded as a positive-negative potential at the cortical surface. Another is that the sum of EPSPs and IPSPs generated in postsynaptic elements extending over the whole depth of the cortex is recorded as the positive-negative sequence wave at the surface. The former explains very well the rapid and smooth transient phase from positive deflection to a negative one. But the idea is not better than a hypothesis, since the activity of the apical dendrite has not yet been revealed with certainty. The latter, though also being difficult to corroborate, explains without contradiction the effects of cortical polarizations on the surface positive-negative potential. While the waxing and waning of the potential in response to low-frequency repetitive stimulation were interpreted by excitability changes in thalamic and cortical neurons. However, phenomena suggesting the mechanisms in the level of cortical neurons were not fully determined.

The series of experiments was planned mainly to clarify the origins of components of the primary and augmenting responses and to discover probable mechanisms of the waxing and waning at neuron level in the augmenting response. Results also allowed to refer to the nature of the recruiting response to some extent.

\section{METHODS}

Experiments were carried out on adult rabbits weighing $2.8-3.5 \mathrm{~kg}$. After anaesthetization with pentobarbital sodium $(20-30 \mathrm{mg} / \mathrm{kg}$. body weight), the trachea was cannulated and paralysed with hexamethylene-1,6-biscalbaminoil choline bromide.

Received for publication December 25, 1965

内田孝 
Artificial respiration was continued throughout the course of experiment. Then the animals were mounted in a stereotaxic instrument and the cortices were exposed by removing the parietal part of the skull just overlying the sensorimotor cortex. Cisternal drainage was also performed to eliminate the brain pulsation accompanying respiration. Cut ends of skin and pressure points caused by head holding were locally anaesthetized with $2 \%$ procaine.

Stimulations of specific and nonspecific thalamic systems were achieved with concentric bipolar silver wires enclosed in pair glass capillaries except the tips (tip diameters 50 micra or less, tip separation $0.5 \mathrm{~mm}$ ). Single, double or $8 \mathrm{c} / \mathrm{s}$ train electroshock was delivered to nucleus ventralis posterolateralis (VPL) in order to set up the so-called primary response or augmenting response in the sensorimotor cortex. To obtain recruiting response originating in nonspecific thalamic nucleus $8 \mathrm{c} / \mathrm{s}$ stimulation was applied to nucleus ventralis anterior (VA). Cortico-spinal tract was also stimulated at the level of pons to identify pyramidal tract cells. These three pairs of stimulating electrodes were held in respective manipulators and placed through the cranial opening with the guide of the SAWYER et al. atlas ${ }^{15}$.

Cortical surface oscillations were recorded from the parietal region of the ipsilateral cortex (sensorimotor cortex) with $0.1 \mathrm{~mm}$ silver wire. This wire was fixed to the inside wall of the small hole drilled in the pressure plate made of acrylic resin, which was designed to prevent the movement of the cortex, and held in a threedimensional manipulator. Micropipettes filled with $3 \mathrm{Mol}$. potassium chloride or $1.5 \mathrm{Mol}$. potassium citrate (5-20 megohms in direct current resistance) were inserted nearly vertically into the cortex close to the surface electrode through the hole of the pressure plate. Microelectrodes were driven with the hydrauric micromanipulator held in an arm of the mechanical manipulator, and depths at which activated cells existed were measured from the contact point of the electrode tip on the pial surface. Exposed cortical surface was covered with Ringer-soaked cotton or Ringer agar after completion of electrode settings. Indifferent silver-silver chloride stick electrode was embedded in the incised neck skin.

In the following figures, the upper trace in each pair sweep was recorded from the cortical surface, while the lower, from the vicinity of an active neuron in the cortex. Positivity is indicated by downward deflection for both surface-electrode and intracortical microelectrode.

\section{RESULTS}

In the course of the experiment many types of cells were encountered in response to the stimulation of thalamic nuclei; that is, about half of the total number examined fired with various latencies in the surface positive-negative phase, and others fired $50-100 \mathrm{msec}$ or more later after the stimulation or replied with the cessation of spontaneous discharges. The present report exclusively deals with the cells which fired during phases of cortical surface waxing and waning sequence, lasting $30-40 \mathrm{msec}$, to the thalamic stimulations. And other units that behaved differently from the above category were omitted, since these units seemed to have no immediate relation to the formation of thalamo-cortical evoked responses.

Recordings of spikes were extracellularly performed upon cortical cells exhibiting the positive or positive-negative spike potential, reaching up to 
$20 \mathrm{mV}$, with a few exceptions intracellularly recorded. In these records extracellular synaptic noises were also picked up and shown as small and irregular ripples on the baseline. This fact would show that the electrode tip was located very close to the soma membrane. In some instances electrodes having a relatively low resistance were used to record the intracortical focal potential together with responding spikes.

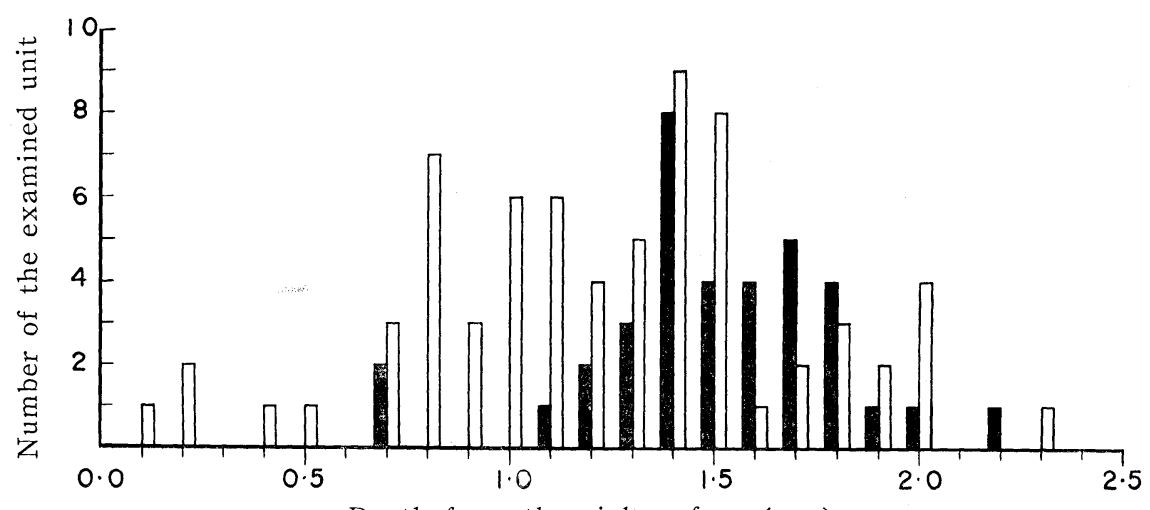

Depth from the pial surface $(\mathrm{mm})$

FIG. 1. Depth distributions of the examined cortical neurons which fired during the surface positive-negative response to the thalamic (VPL) stimulation. Black columns, pyramidal tract cells; white columns, non-pyramidal tract cells.

Of 108 neurons examined, about two-thirds were non-pyramidal tract cells (non-PT cells), almost all of the rest were pyramidal tract cells (PT cells), and only three cortico-thalamic cells. Depth distributions in the cortex of PT cells and non-PT cells are shown in FIG. 1. Although non-PT cells existed over practically all depths, those which responded with fairly short latency to the VPL stimulation were often encountered at depths below $0.7 \mathrm{~mm}$ from the cortical surface. PT cells were situated in rather confined cortical levels ranging from 1.2 to $1.8 \mathrm{~mm}$.

Variations of the response pattern in non-PT cells. In firing characteristics there were many types of non-PT cells responding during evoked potentials to single or repetitive VPL stimulation, but they could be classified into several patterns in responding manner as described below. Some neurons fired to single VPL stimulation and others to repeatedly applied ones. This difference would not be substantial, because the latter might have more direct relation to the afferents from the other thalamic relay nucleus.

In FIG. 2 two examples are illustrated which responded to single thalamic shock. One of them fired to a single stimulus with a short latency, about 3 msec in the shortest case, corresponding to the beginning of the positive phase in the surface monitoring potentials in A. To the successive stimulus 

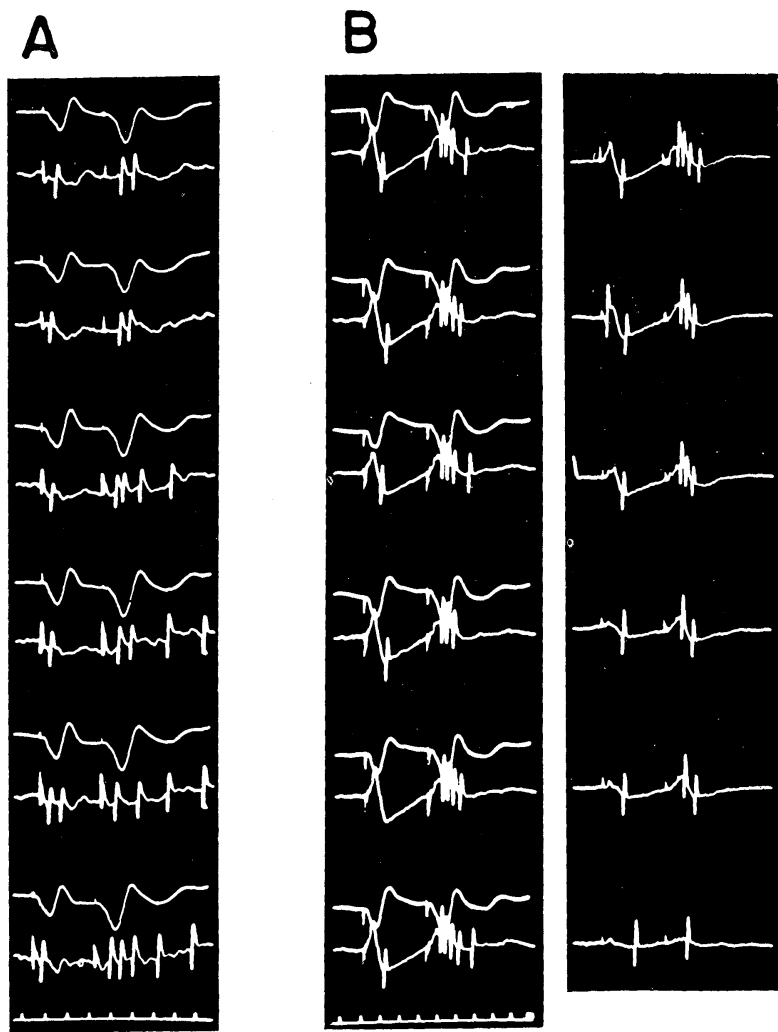

FIG. 2. Effects of paired stimuli on two cells responding to single VPL stimulation. A : Second stimuli delivered at $30 \mathrm{msec}$ interval delays the latency, increases the firing numbers, and makes higher the amplitude of the surface response. Stimulus strengths increase gradually from upside downwards. Depth, $0.9 \mathrm{~mm}$. B : Second stimulus has the same effects on the cell as in A, except decrease in latency and massive firings. In the left column stimulations of the same strength were given in six pair sweeps in order to test the reappearing nature of the effect, while gradual decrease in stimulus strength in the right column (only intracortical records were shown). Depth, $1.3 \mathrm{~mm}$; time marks, $100 \mathrm{c} / \mathrm{s}$.

given after $30 \mathrm{msec}$, this call responded with an increased latency, 6-8 msec, of the initial spike in the train discharges. Another cell in B, firing with a long latency about $11 \mathrm{msec}$ and in the surface negative phase to single shock, started to respond with a decreased latency up to $7 \mathrm{msec}$ to the second stimulus given at $33 \mathrm{msec}$ interval. There is a reverse relation with respect to the latency shift in the two cells in FIG. 2A and B. These transitions in firing nature were also observed in low-frequency VPL stimulation $(8 \mathrm{c} / \mathrm{s})$.

In spite of the above-mentioned difference, some common features are noticed between the two, i. e. to the second and subsequent stimulations, both cells respond with two or more spike discharges-variable in number with 
the stimulus strength and the rate of repetition-, and tend to establish a constant latency and firing phase, and also the increase in spike number is associated closely with the augmentation in the surface positive potential.

Many cells respond more or less with variable latencies depending upon the stimulus strength, shorter with increasing strength. Fig. 3A is the case most conspicuous in this respect. This cell did not fire with a weak single
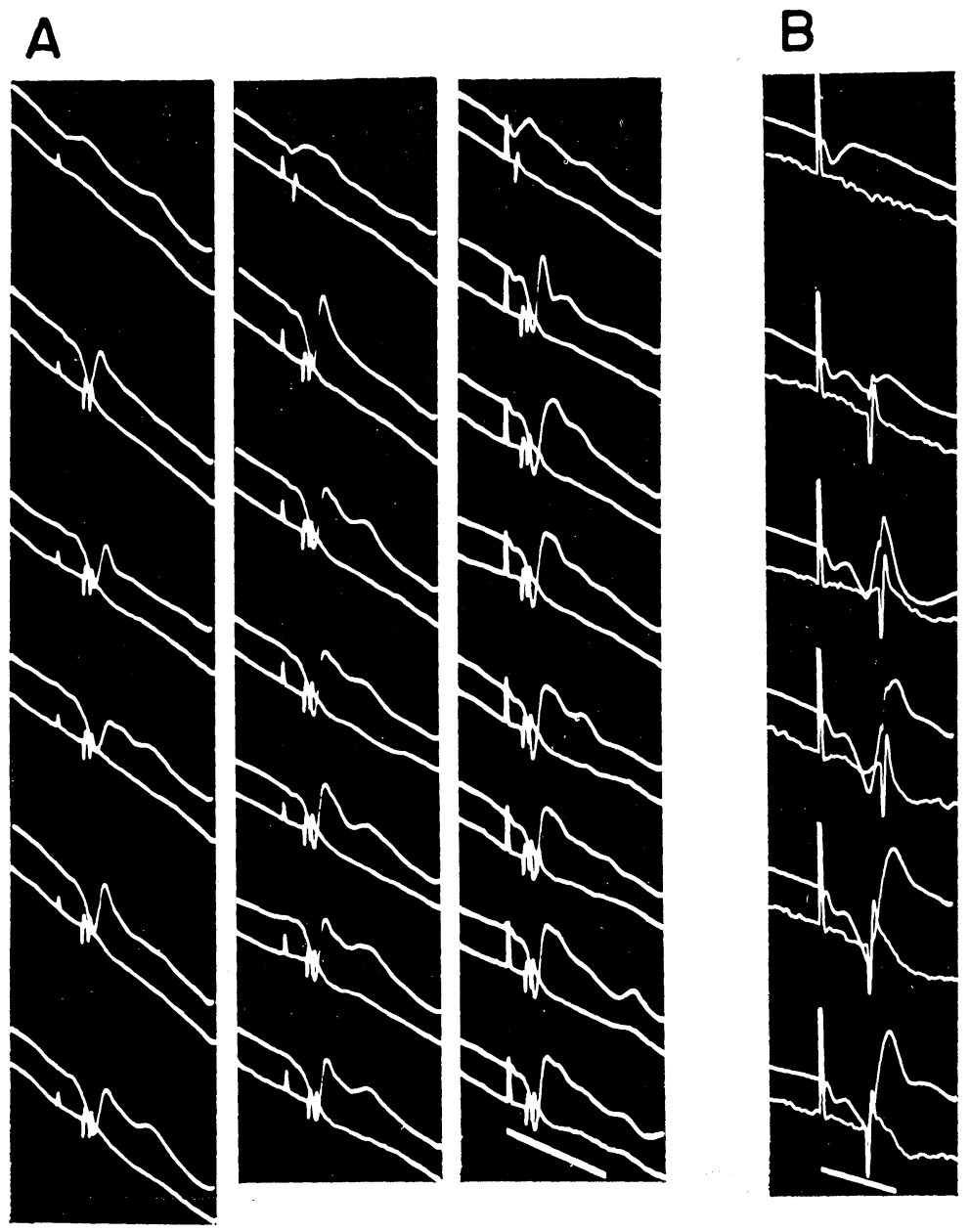

FIG. 3. Activities of cells to $8 \mathrm{c} / \mathrm{s}$ VPL stimulation. A: The cell fired at second shock with weak stimuli (left column), while stronger stimulation elicited a spike during the initial positive deflection at first shock (middle, two-times stronger than left; right, four-times) and double spikes during the augmenting positive phase at second and subsequent shocks. Increasing the strength reduces the latency for firing. Depth, $1.4 \mathrm{~mm}$; time bar, $50 \mathrm{msec}$. B: Another cell showing gradual decrease in latency and fixation of the latency at the peak of the augmenting surface positive wave to $8 \mathrm{c} / \mathrm{s}$ VPL stimulation. Depth, $1.5 \mathrm{~mm}$; time bar, $20 \mathrm{msec}$. 
stimulus, but fired with a relatively short latency to a strong one and with a lengthened latency to successive ones, like the cell in Fig. 2A. As shown in the figure, the cell fired singly in the initial positive deflection to the first stimulus, and also fired in duplet concentrating upon the augmenting positive potential to successive $8 \mathrm{c} / \mathrm{s}$ stimuli. Latencies for the spike initiation were shortened with the increasing of the stimulus strength, but the latency in a given strength remained nearly unchanged during $8 \mathrm{c} / \mathrm{s}$ repetitive stimulation. Another type of cortical neurons is shown in FIG. 3B. This cell initially fired in the surface negative phase, not yet fully developed. But repetition of VPL stimulation caused gradual reduction in latency and finally brought the latency to the peak of the augmenting surface positive deflection. This type of cells was met most frequently and occupied about half of the total non-PT cells in this experiment.

Augmenting response begins to decrease in amplitude after attaining the development of maximal size, generally on or after the tenth to fifteenth response. Relations between spike firing and surface response were also examined in this waning phase. The cell, shown in FIG. 4A, responded with four to five spikes during development of maximal phase of augmentation, until the ninth stimulus; however, further repetition of the stimulation led to the decrease in spike numbers in coincidence with diminution of the surface response, especially of the positive potential. While the latency of the first spike in the train discharges held a constancy throughout the series of the waxing and waning, the firings were temporally concentrated on the augmenting surfacepositive components during the waning phase. Some cells which responded with single spikes during the waxing and fully-developed phases failed to fire during the waning phase. One of these cells is illustrated in Fig. 4B. This record was taken intracellularly from the cell which was exhibiting relatively low membrane potential and then thought to be drawing near an injury state. There are shown first five responses superimposed upon each other to $8 \mathrm{c} / \mathrm{s}$ stimulation of VPL. In lower records single spikes rose from the increasing depolarization potential (EPSPs), downward deflection, with a stable latency in response to the second and subsequent stimulation and was followed by a gradually growing hyperpolarization (IPSPS). Increase of the EPSPs to repetitive stimulation was not so distinct, probably owing to the lowered membrane potential by impaling the cell, while growing of the IPSPs after firing was powerful and reached about $20 \mathrm{mV}$. Repetitive stimulations reduced the latency of the full-grown IPSPs during the waning phase of the surface response, accordingly the preceding EPSPs were nibbled and lowered by the IPSPs resulting in failure of further spike production, though not shown in the figure. EPSPs and IPSPs in the place were temporally related to the surface-positive and -negative deflections, respectively.

There were some neurons which discharged sustained trains of impulses 
A

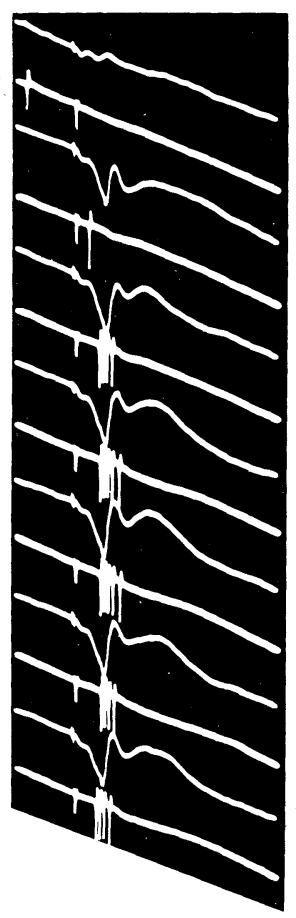

B

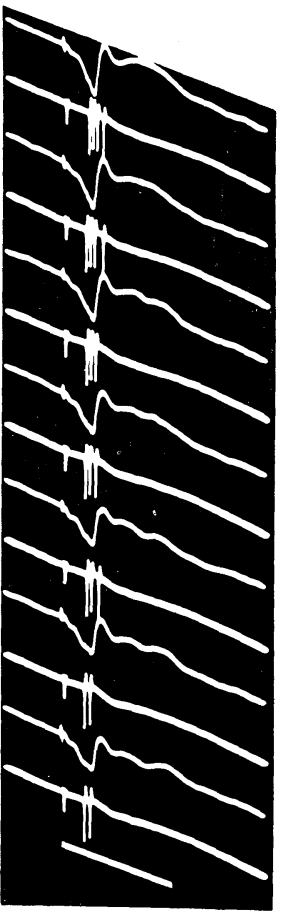

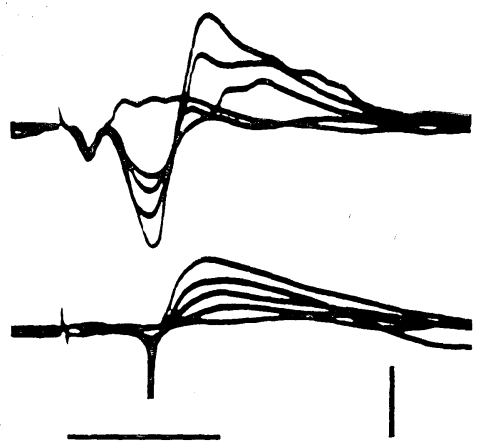

FIG. 4. A : Cell activity during waxing and waning to $8 \mathrm{c} / \mathrm{s}$ VPL stimulation. Firing numbers, increased up to four to five during maximum augmenting phase, decrease in the later phase. The decrease in firing corresponds with the decrease in amplitude of the augmenting positive component. Depth, $2.2 \mathrm{~mm}$; time bar, 50 msec. B: Relation between intracellular PSPs and firing in a cell (superimposed records of first five responses to $8 \mathrm{c} / \mathrm{s}$ VPL stimulation). Repetitive stimulation grows not only EPSPs in the cell from which single spikes rise, but also IPSPs which decrease gradually in latency, then nibbles the preceding EPSPs, and inhibits to fire at last. About two-thirds of the spike tip was cut away from the print. Depth, $1.5 \mathrm{~mm}$; time bar, $20 \mathrm{msec}$; vertical bar, $20 \mathrm{mV}$ for lower records only.

at frequencies of $400-500 \mathrm{c} / \mathrm{s}$, generally exhibiting a small deflection, to the repetitive VPL stimulation $(8 \mathrm{c} / \mathrm{s})$. These are considered to originate from interneurons similar to those demonstrated in the spinal cord, although the exact criterion for identification has not been established in the cerebral cortex. FIG. 5A illustrates the responding fashion of an example of such cells. The cell fired several times sporadically to the first stimulus; to subsequent stimuli, however, it responded with a massive impulse train which declined gradually in frequency and lasted about $50 \mathrm{msec}$. The period for which discharges lasted covered the whole phase of the long-lasting negative potential during development of maximal phase of augmentation. Although 


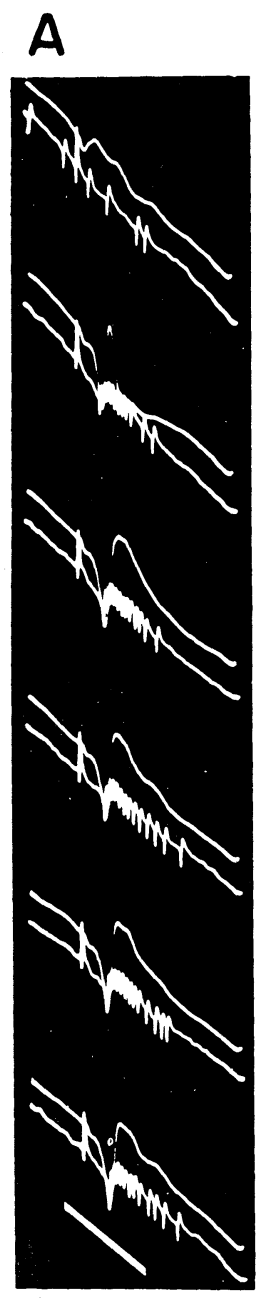

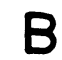

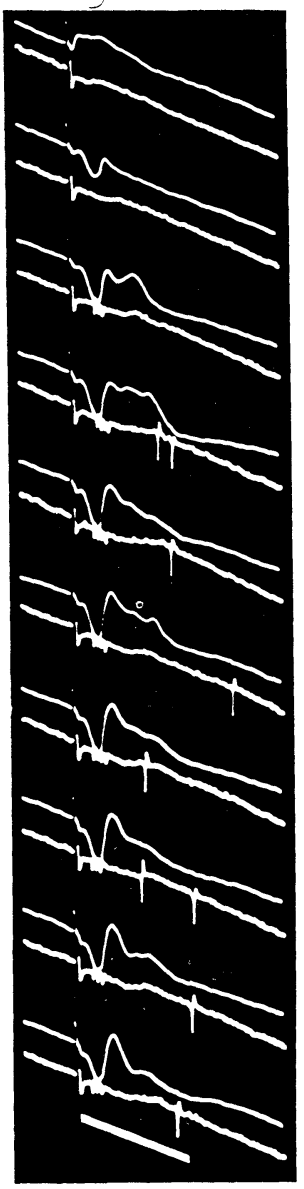

FIG. 5. Activities of cells thought to be interneurons to $8 \mathrm{c} / \mathrm{s}$ VPL stimulation. $\mathrm{A}$ : Second and subsequent stimuli elicite repetitive spike train reaching to $500 \mathrm{c} / \mathrm{s}$ in frequency and continuing about $50 \mathrm{msec}$. Depth, $0.4 \mathrm{~mm}$; time bar, $50 \mathrm{msec}$. B : Third and later stimuli initiate repetitive train discharges in the phases of the augmenting components. Latency of the first spike in each train decreases gradually. Larger spike in later phases is from another cell situated nearby. Depth, $2.3 \mathrm{~mm}$; time bar, $50 \mathrm{msec}$.

in this cell a latency shift was not observed among the first spikes of each train during augmentation, there was another cell which showed a gradual reduction in the latency of the first spike from $11 \mathrm{msec}$ to $7 \mathrm{msec}$ (FIG. 5B, small deflections in the surface positive phase). It is possible that some cells of this type act on other cortical neurons as illustrated in FIG. 4B, and develop long-lasting IPSPs in these cells, resulting at last in failure of firings during 
the waning phase in the surface record of repetitive thalamo-cortical responses.

Activities in non-PT cells during recruiting response. Repetitive VA stimulations at $8 \mathrm{c} / \mathrm{s}$ have produced the waxing and waning positive-negative potentials so-called recruiting response in the sensorimotor cortex. These waves recorded at cortical surface differ from the augmenting responses obtained by stimulating VPL in that these have longer latencies and are lacking in initial -small positive deflections which occur in a few msec after stimulation and precede the positive-negative potentials in the augmenting response.

About two-thirds of the cells that responded to the VPL stimulation also fired in the various phases of recruiting response to the repetitive VA stimulation. Some cells fired in the surface positive phase with a fairly steady latency similar to the response to the VPL stimulation. An example of such cells is illustrated in FIG. $6 \mathrm{~A}$ and $\mathrm{B}$. To the $8 \mathrm{c} / \mathrm{s}$ VPL stimulation the cell fired at the peak of surface-positive deflection (corresponding to the negative phase of intracortical field potential) with the latency of $13.5 \mathrm{msec}$ (FIG. 6A). The same cell also responded to $8 \mathrm{c} / \mathrm{s}$ VA stimulation at the surface-positive phase with a longer latency $(22-24 \mathrm{msec})$ than in the response to VPL stimulation (FIG. 6B). Large numbers of the cells activated by both VPL and VA stimulation fired in the surface-negative or the delayed negative phases to VA stimulation; therefore the latencies for spike firing were longer than that in the above-mentioned instance (FIG. 6C).

General characteristics of the responses to VA stimulation differing from those to VPL stimulation are as follows. As has been expected from the fact that the latency of surface-nonspecific response is fairly longer than that of the specific response, the latency for firing in a given cell is also evidently longer than that to the VPL stimulation. Through a series of recruiting response, latencies are more variable than in augmenting sequences and fluctuations in latency are enormous in cells which respond with longer latencies. Cells such as those responding to single stimulus or to the first of the repetitive train stimuli applied to VA are rare and there are frequent lacks of spike firing even during stabilization phases of recruiting responses. Neurons firing in the surface-positive phase of recruiting response occupied a small rate in number as compared with those that responded in the same phase of responses to VPL stimulation. A few neurons responded in the surface-positive or -negative phase of recruiting response only to VA stimulation.

Responses of PT cells to VPL and VA stimulations. Most all PT cells responded to single or repetitive VPL stimulation which was the orthodromic stimulation for these cells. Roughly speaking, PT cells resemble non-PT cells in responding manners to VPL stimulation. One-third of PT cells studied fired before or at the peak of the augmenting surface-positive potential (FIG. 

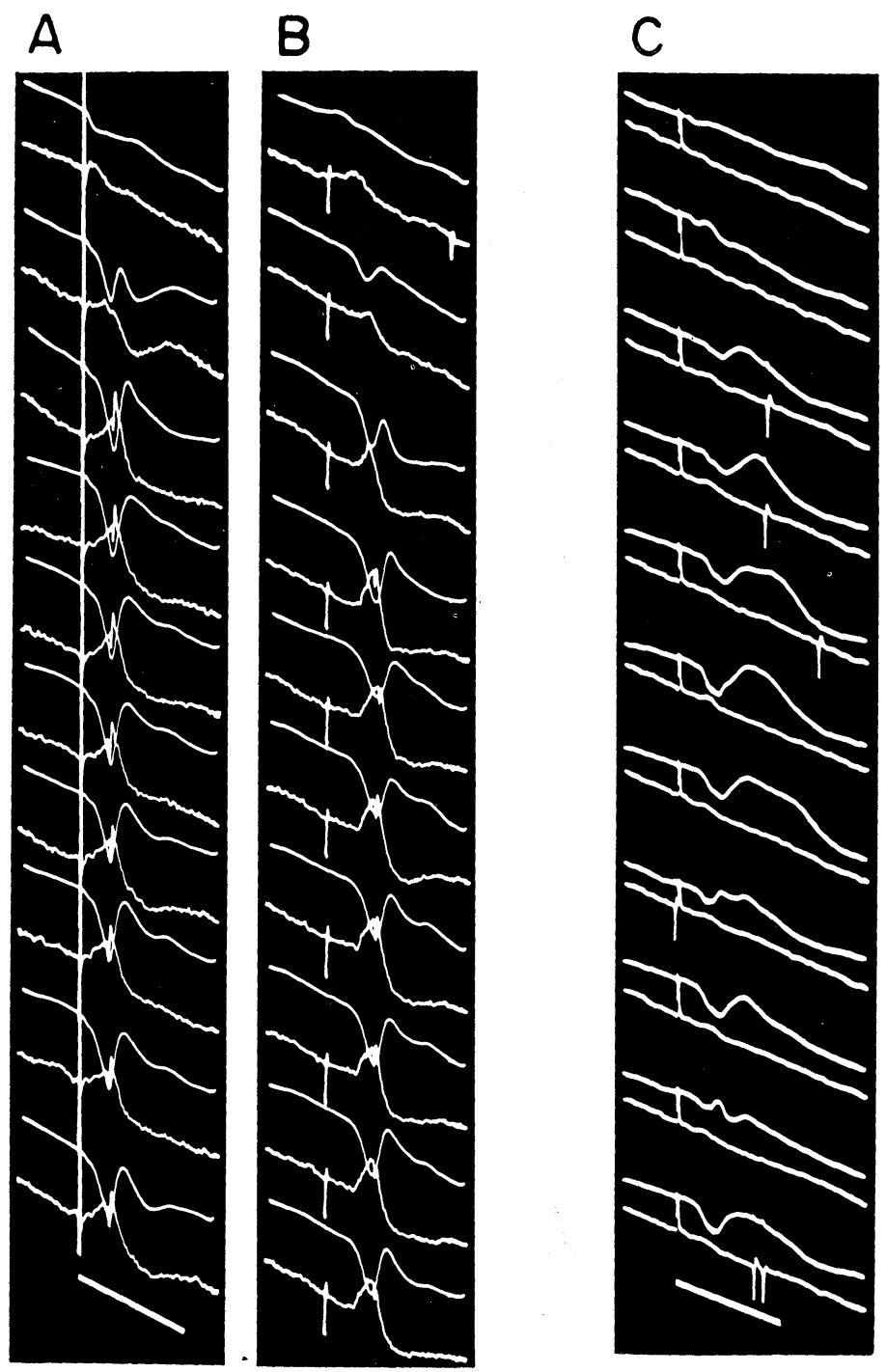

Fig. 6. Activities in non-PT cells to $8 \mathrm{c} / \mathrm{s}$ VA stimulation. A, B: The cell responding at the peak of the augmenting surface positive potential to VPL stimulation (A) also fires in the surface positive phase in the recruiting response caused by VA stimulation (B). Spike height has been reduced by a slight movement of the electrode in the experimental course. Depth, $1.1 \mathrm{~mm}$; time bar, 50 msec for A and B. C: A cell responded rather sporadically in the surface negative potential to VA stimulation. Depth, $1.6 \mathrm{~mm}$; time bar, $50 \mathrm{msec}$.

$7 \mathrm{~B}$ ), one half in the transient phase going negativity (going-negative phase) or the negative phase (FIG. $7 \mathrm{C}$ ), and the rest in the delayed surface negative wave to repetitive VPL stimulation. 

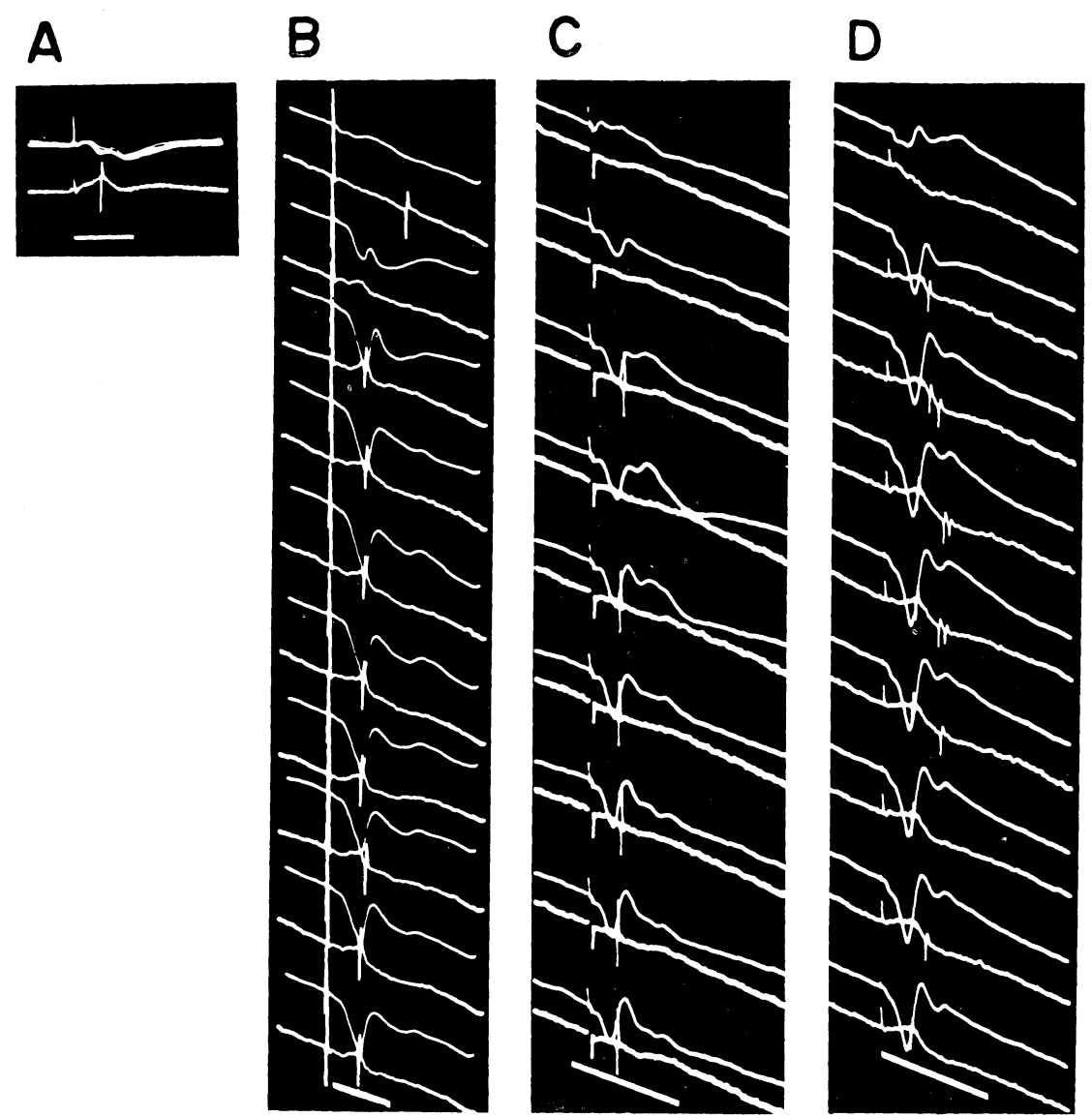

Fig. 7. Activities of PT cells to VPL stimulation. A: A PT cell firing to single VPL stimulation (superimposed records of five sweeps at $1 \mathrm{c} / \mathrm{s}$ ). Depth, 1.4 $\mathrm{mm}$; time bar, $30 \mathrm{msec}$. B: The one firing at the peak of the augmenting surface positive deflection. Depth, $1.4 \mathrm{~mm}$; time bar, $25 \mathrm{msec}$. C: The one firing during the going-negative phase. Depth, $1.6 \mathrm{~mm}$; time bar, $50 \mathrm{msec}$. D: The one firing during the going-negative, negative, and delayed negative phases. Depth, $1.2 \mathrm{~mm}$; time bar, $50 \mathrm{msec}$.

An example of PT cells which fired in response to single VPL stimulation is shown in Fig. 7A, where five sweeps were consecutively superimposed. There were small numbers of such cells as shown in this figure and many began to respond at the second to third stimulus in the train $(8 \mathrm{c} / \mathrm{s})$ of VPL stimulation. FIG. 7B is one of the PT cells which responded in the peak of surface-positive phase, and FIG. 7C shows one that responded in the goingnegative phase of the surface response. Less fluctuation and shift in the latency for firing are recognized in both neurons as compared with non-PT cells during augmentation. Another type of PT cell is illustrated in FIG. 7D. 
This cell fired in the going-negative phase or the peak of the negative potential and/or the delayed negative potential, sometimes in duplet, in the surface response during repetitive VPL stimulation. Such a cell is rare in number throughout this experiment.

Main features of PT cell responses to VPL stimulation can be summarized as follows. PT cells which respond to single VPL stimulation or respond with plural firings are less than those in non-PT cells. The reduction and fluctua-
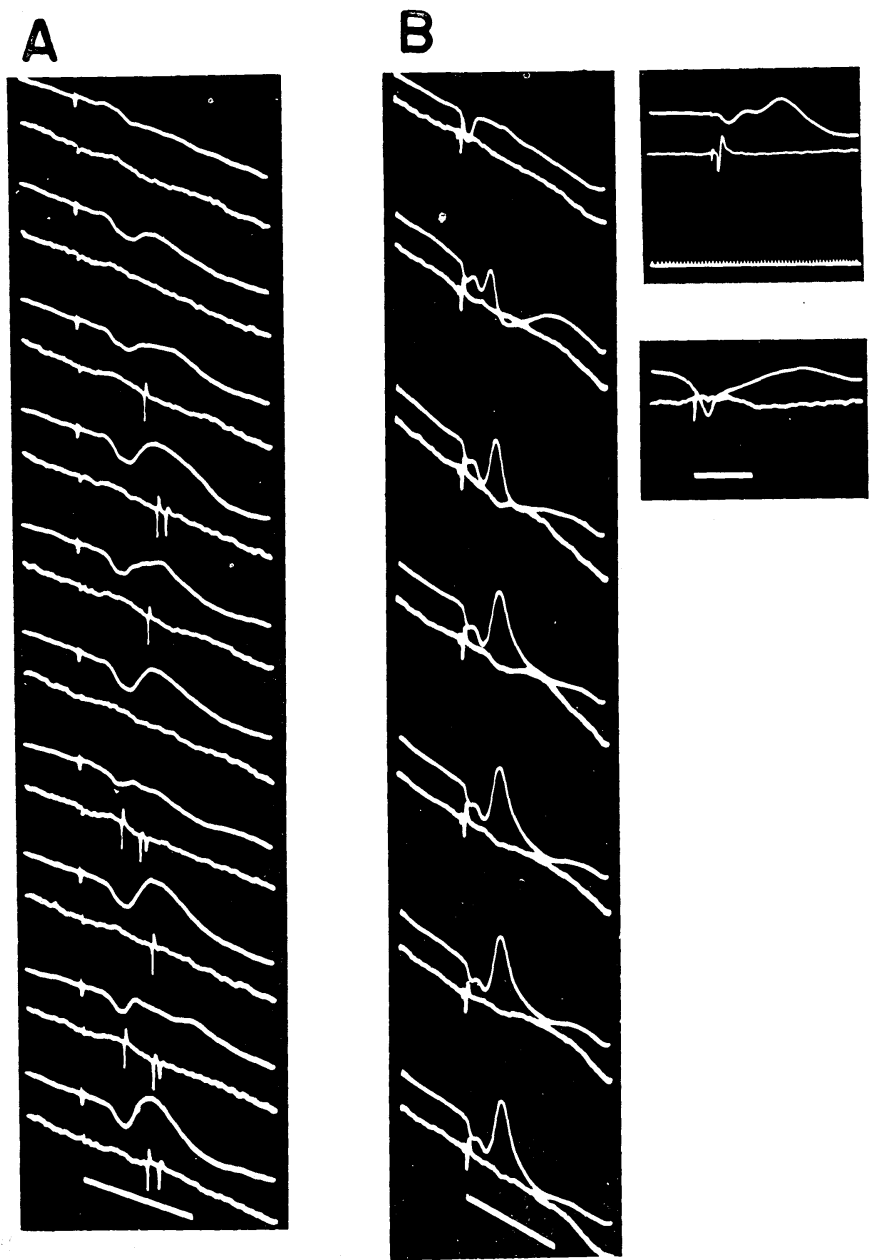

FIG. 8. A : Activity in a PT cell to $8 \mathrm{c} / \mathrm{s}$ VA stimulation. The same PT cell as in FIG. 7D fires with fluctuated latency in the surface positive and negative phases. Time bar, $50 \mathrm{msec}$. B: Cortico-thalamic cell responding with a constant and short latency to $8 \mathrm{c} / \mathrm{s}$ VPL (left column) or single VPL (right upper) stimulation. This cell also fires in the early phases of spontaneous cortical spindle waves (right-lower). Depth, $2.2 \mathrm{~mm}$; time marks, $50 \mathrm{msec}$ for left column, $1000 \mathrm{c} / \mathrm{s}$ for upper-right, and $30 \mathrm{msec}$ for right-lower. 
tion in latency are generally not recognizable during $8 \mathrm{c} / \mathrm{s}$ repetitive activation of PT cells. The cells firing in the going-negative or negative phase of the surface response are most frequently encountered, while in non-PT cells ones responding before or at the peak of surface positive potential are the most.

VA stimulation could activate a small number of PT cells suggesting that VA neurons scarcely have relation with cortical PT cells. A PT cell shown in FIG. 8A, the same cell as in FIG. 7D, fired singly or plurally with fairly fluctuated latency in the surface positive and/or negative phases during fullgrown recruiting response in response to $8 \mathrm{c} / \mathrm{s} \mathrm{VA}$ stimulation.

Antidromic activation of cortico-thalamic neurons. In the whole course of experiments, a few neurons were met which fired by the peak time of the initial positive deflection in the surface response to VPL stimulation. This type of the cortical cells has a steady threshold for firing and a short and constant latency regardless of the strength of stimulations applied singly or repeatedly to VPL. In addition the spike potential of such a cell was always accompanied with synaptic noises, suggesting that the activity was recorded from the extreme vicinity of soma membrane of a cell. These facts mean that the action potential was not caused transsynaptically in the cortical neuron, nor recorded from a terminal portion of the afferent projection fiber from the thalamus. Therefore, the firing should be the antidromic activation of a cortico-thalamic cell which sends its axon to VPL, or of another cell which sends the axon collateral to this nucleus. To a single or repetitive stimulation of VPL the cell shown in FIG. 8B fired singly with a constant latency of about $1.5 \mathrm{msec}$ at the onset of the initial positive phase of the surface response. This cell also fired in the early phases of spontaneous cortical spindle waves (FIG. 8B, right-lower). There was another corticothalamic neuron which also responded orthodromically with one to three spikes in the augmenting surface positive phase after the antidromic activation.

\section{DISCUSSION}

Components of evoked potentials. Evoked potentials obtained in the sensorimotor cortex to thalamic stimulations of the rabbit resemble in many respects those recorded in the cat. In the so-called primary response evoked by VPL stimulation the initial positive deflection is most distinct. This deflection appears solely (cf. FIG. 8B), accompanied by successive small negative potential (FIG. 7C) or slower positive potential (FIG. 6A), or sometimes followed by a germination of the augmenting positive-negative wave (FIG. 4A) which usually manifests to repetitive stimulation. These variations in wave form were empirically understood to be ascribable to the differences in the intra- 
nuclear stimulating point and more largely in the cortical recording site, and were identical to the observations in the cat.

The initial positive deflection of the primary response has been stated to disappear with low-frequency repetitive stimulation ${ }^{5,6}$. But, in this experiment this component remained the same, or became reduced in height with $8 \mathrm{c} / \mathrm{s}$ VPL stimulation, as shown by CLARE and BISHOP). While the augmenting positive-negative response grows gradually with successive stimuli and attains maximum amplitude at certain fifth stimulus. When the latency of the positive wave is longer, this wave and the initial positive deflection are obtained independently. Usually, however, both phases are recorded as a two-step positive wave which has an inflection on the time course. The variability in latency of the augmenting component is considered to be dependent on differences in the recording site cf.11). $^{\text {. }}$

Since the initial positive deflection appears with a very short latency, activities in the terminal portions of the thalamo-cortical projection fibers must take a part in the formation of the deflection. The cortico-thalamic cells also fired antidromically in this phase. In addition to these, postsynaptic activities of some cortical neurons which respond with short latencies are involved in this deflection. Postsynaptic neurons firing in this phase are small in number. These fired in the initial positive phase only to the initial (or rarely second) stimulus and started to respond in the later augmenting positive phase to subsequent ones of $8 \mathrm{c} / \mathrm{s}$ train stimuli (FIG. 3A). Decrement in amplitude or disappearance of the initial positive deflection to the lowfrequency thalamic stimulation would be caused by such cells as shifting in latency. As this potential reversed in sign into negative below $0.6 \mathrm{~mm}$ from the cortical surface and attained maximal amplitude at the depth of $0.9-1.2$ $\mathrm{mm}$, the initial positive deflection recorded at cortical surface would show the reflection of the intracortical negative field potential due to the activities in arborized afferent terminals and EPSPs generated in some of cortical neurons as mentioned above.

It is thought reasonable that the activities of slower afferents participate in the augmenting positive wave. Postsynaptic activations of cortical cells, however, should play a more important role in the formation of the positive potential. In fact, many cortical cells including PT cells have fired massively concentrated in this positive phase during the full-grown augmenting phase. This potential turned into negative in sign when recording at $0.6-1.5 \mathrm{~mm}$ below the cortical surface and massive spike discharges superimposed on this negative potential. The intracortical negative potential also augments proportionally to the surface positive wave. EPSPs were demonstrated to continue during this surface positive phase in some intracellular recordings. These facts strongly suggest that EPSPs elicited in respective postsynaptic cortical neuron, synchronize at spike discharging and make an intensive ex- 
tracellular negative field deep in the cortex during the augmenting phase, and the negative field potential reflects as a positive wave to the cortical surface.

While PURPURA et $a .^{12,13)}$ reported that in many cells EPSPs and IPSPs are recorded in different cells during different components, and that there is no direct correlation between intracellular PSPs and different phases of cortical surface response. Supposing, however, that the intracortical negative potential is the algebraic sum of negative-positive field potentials which resulted from respective EPSP- and/or IPSP-producing cell, the short duration of the potential is evaluated without contradiction. With this in mind, the surface negative wave can be explained as the reflection of the intracortical positive field potential originated from IPSPs which are usually generated later than EPSPs. Since the deep positive potential, however, is less conspicuous than the preceding negative deflection, different activities in the cortex may take more important parts in the augmenting negative component. They are probably PSPs (especially EPSPs) generated in elements of upper layers, as has been suggested by PURPurA et al. ${ }^{13)}$. Postsynaptic activities in upper layers may also compose the delayed surface negativity. The explanation that evoked potentials recorded in cortical surface are mainly the manifestation of compound postsynaptic potentials is supported by the fact that the membrane potential change, caused by the cortical polarization, changes the amplitude of intracellular PSPs and these reflect directly on different component potentials of surface evoked potentials ${ }^{14}$. These results and considerations are incompatible with another concept that the surface negative potential results from the upward conduction of the depolarization potential along apical dendrites $2,3,9,16)$.

About two-thirds of cortical neurons except PT cells activated by the VPL stimulation also responded to repetitive VA stimulation. It is certain, therefore, that these cells play a role in the production of recruiting response. Some types of these cells fired in the surface positive phase and the others in the surface negative phase with fairly long and fluctuated latency. This would mean that projection fibers from VA are small in number and less synchronized volleys of impulses impinge upon the cortical neurons through multisynaptic and variable pathways ${ }^{10)}$. The fact that the surface negative potential is relatively larger in amplitude than the preceding positive one would suggest the afferents from VA mostly terminate on the cortical elements in upper layers and the massive negative field due to PSPs, especially EPSPs, in upper layers overwhelms the reflected positive potential from activities in deep elements. At all events, more complicated intracortical pathways must be involved in recruiting response than in the augmenting response.

Probable mechanisms of the waxing and waning in the augmenting response. About a half of the cortical cells encountered fired in the course of the 
surface positive-negative phase in response to single or repetitive stimulation applied to VPL. Behaviors of these cells to low-frequency $(8 \mathrm{c} / \mathrm{s})$ repetitive stimulation are as follows; (a) cells, not eliciting spike to single shock, start to fire in the augmenting surface positive phase to successive stimuli, (b) cells, initially responding with single spikes, fire plurally to repetitive stimulation, (c) latencies of some cells are delayed down to the augmenting positive phase to second and subsequent stimuli, (d) latencies of other cells are shortened gradually and at last fix at the peak of the augmenting positive phase with repetition of stimulation. These phenomena show that activated cells increase in number and the timings of their firing synchronize in the augmenting positive phase. As mentioned previously, the deep negative field potential due to EPSPs augments gradually during repetitive VPL stimulation and reflects as the augmenting positive wave to the cortical surface. The mechanism by which (a) and (b) take place is thought to be that the increase in activated afferent fibers leads to the augmentation of EPSPs generated in respective cortical neuron, as the preceding shock raises the excitability of thalamic elements. Delaying in latency (c) might be brought about by the change in thalamic mediating neurons into slower projection cells owing to the activity of intrathalamic or thalamo-cortical reverberating system. While the gradual shortening in latency (d) might mean that the rising of EPSPs becomes rapid or intermediate neurons gradually decrease in number due to activation of cortical excitatory interneurons.

During waning phase in the augmenting response intracortical deep negative potential decreases in amplitude corresponding to the surface positive wave. At this time gradual decrease in firing number, cessation of firing, or fluctuation in latency take place. Waning in cortical slow potentials is certainly caused by the decrease of the intracortical field potential arising from the decrease of EPSPs in respective neuron and from desynchronized synaptic activities. In some neurons the decrease in EPSPs in fact led to the cessation of spike firing. In the example shown in FIG. 4B, IPSPs which occurred later than EPSPs augmented gradually, decreased in latency, and nibbled the preceding EPSPs resulting in failure of firing. The increasing IPSPs in a given neuron are probably brought about by activities of cortical inhibitory interneurons resemble to those demonstrated in the thalamus ${ }^{1}$.

While the mechanism of the waxing and waning in the recruiting response may be a more complicated one than in the augmenting responses, since subcortical, cortical, and thalamo-cortical reverberating mechanisms are thought to be intermingled for the manifestation of recruiting response ${ }^{\text {cf. } 8 \text { ) }}$. However, facts that there are common cortical neurons responding to both VPL and VA stimulations and interactions are recognized between the slow components of the augmenting and recruiting responses ${ }^{\text {e.g. }}$. $)$, might show that the same mechanism of the waxing and waning as in the augmenting re- 
sponse also participate in those in the recruiting response.

\section{SUMMARY}

1. Interrelations between the slow potential sequence and neuron activities in the sensorimotor cortex to the stimulations of thalamic relay (VPL) and nonspecific (VA) nuclei were studied in the rabbit and the results led to the following considerations.

2. The initial positive deflection recorded at the cortical surface is the reflection of the intracortical negative potential which resulted from massive terminal activities in the fastest thalamo-cortical projection fibers and synaptic activities in some cortical neurons firing with short latency and adapting in a short time.

3. The augmenting positive-negative potential is brought about by the deep negative-positive field potential which results from EPSPs and IPSPs generated in many cortical cells and reflects upon the cortex in the reverse sign. In addition, postsynaptic activities in upper layers must participate in the formation of this potential, especially of the negative phase.

4. Gradual synchronization of PSPs produced in respective neuron makes the intracortical field potential larger when stimulated at $8 \mathrm{c} / \mathrm{s}$. While IPSPs developing later, probably owing to the activities of inhibitory interneurons, lower the height of EPSPs and lead to the inhibition or cessation of firings. These are possible mechanisms by which the waxing and waning occur in response to low-frequency VPL stimulation.

5. Less evidence was obtained to allow making the distinct relation between neuron activities and cortical slow potentials to $8 \mathrm{c} / \mathrm{s}$ VA stimulation. Therefore, strict ideas on the origins of component potentials in the recruiting response were not obtained. It is reasonable, however, to presume that the same elements and mechanisms as in the augmenting resfcrse also take a part in the recruiting response.

The author wishes to express his sincere thanks to Prof. Y. IwASE for his invaluable discussion and criticism. He also thanks to Dr. K. MURAYAMA for his help in the later stage of the experiment and to Brother A. T. PION for his help in preparing the manuscript.

\section{REFERENCES}

1) Andersen, P. And Sears, T.A. The role of inhibition in the phasing of spontaneous thalamo-cortical discharge. J. Physiol., 173: 459-480, 1964.

2) Bishop, G.H. The dendrite: Receptive pole of the neurone: In A Symposium on Dendrites. Electroenceph. clin. Neurophysiol., Suppl. 10, pp. 12-21, 1958.

3) Clare, M. H. And Bishop, G.H. Properties of dendrite: apical dendrites of cat cortex. Electroenceph. clin. Neurophysiol., $7:$ 85-98, 1955. 
4) Clare, M. H. and Bishop, G. H. Potential wave mechanism in cat cortex. Electroenceph. clin. Neurophysiol., $8:$ 583-602, 1956.

5) Dempsey, E.W. And MoRison, R.S. The production of rhythmically recurrent cortical potentials after localized thalamic stimulation. Am. J. Physiol., 135: 293300, 1942.

6) Dempsey, E.W. And Morison, R.S. The mechanism of thalamo-cortical augmentation and repetition. Am. J. Physiol., 138: 297-308, 1942.

7) Jasper, H. H. And Ajmone Marsen, C. Thalamo-cortical integrating mechanism. Res. Publ. Ass. nerv. ment. Dis., 30: 493-512, 1951.

8) JASPER, H.H. Unspecific thalamo-cortical relations: In Handbook of Physiology edited by J. Field, Am. Physiol. Soc., 2 : 1307-1321, 1960.

9) LI, C.-L. Cortical intracellular synaptic potentials in response to thalamic stimulation. J. cell. comp. Physiol., 61: 165-179, 1963.

10) Morillo, A. Microelectrode analysis of some functional characteristics interrelationships of specific, association and non-specific thalamo-cortical systems. Electroenceph. clin. Neurophysiol., 13: 9-20, 1961.

11) Morse, R.W. And Towe, A.L. The dual nature of the lemnisco-cortical afferent system in the cat. J. Physiol., 171: 231-246, 1964.

12) Purpura, D. P. And Shofer, R. J. Cortical intracellular potentials during augmenting and recruiting responses. I. Effects of injected hyperpolarizing currents on evoked membrane potential changes. J. Neurophysiol., 27 : 117-132, 1964.

13) Purpura, D. P., Shofer, R. J. And Musgrave, F. S. Cortical intracellular potentials during augmenting and recruiting responses. II. Patterns of synaptic activities in pyramidal and nonpyramidal tract neurons. J. Neurophysiol., $27: 133-151$, 1964.

14) Purpura, D. P. And McMurtry, J. G. Intracellular activities and evoked potential changes during polarization of motor cortex. J. Neurophysiol., $28: 166-185$, 1965.

15) Sawyer, C.H., Everett, J. E. And Green, J.D. The rabbit diencephalon in stereotaxic coordinates. J. comp. Neurol., 101: 801-824, 1954.

16) Spencer, W. A. And Brookhart, J. M. Electrical patterns of augmenting and recruiting waves in depths of sensorimotor cortex of cat. J. Neurophysiol., 24: 26-48, 1961. 